\title{
From Snowy White Peaks to Angels of Colour -
}

Ingrained Differential Outcomes of COVID-19 for a Diverse Health Workforce

Indranil Chakravorty PhD FRCP

Vipin Zamvar MS FRCS(Cth)

@

indranil.chakravorty@nhs.net

@

zamvarv@hotmail.com

Keywords: BAME, Risk stratification, COVID-19,

cité Chakravorty, I. \& Zamvar, V. (2020) From snowy white peaks to angels of colour: Ingrained differential outcomes of COVID-19 for a diverse health workforce. The Physician 6(1) epub 24.05.2020 DOI: $10.38192 / 1.6 .1 .12$

\section{Editorial}

The COVID-19 pandemic has profoundly changed the world. In an incredibly short space of time, it has demonstrated the value of international preparedness, the coordination beyond borders from United Nations agencies (such as the World Health Organisation), the politics of leadership and science, with a potential to shape the destiny of billions of people across the world. We see scientists coming together, sharing and collaborating their emerging knowledge, freely, to help nations/communities, who have disparate challenges in combating the virus. As the pandemic ravages through continents, we also see how the variations in the political interpretation of 'science' can affect the destinies of different peoples. Compared to China and South Korea, the experience in societies with large proportions of migrant populations, has revealed a perhaps unpleasant angle to this pandemic, one where there are differential outcomes within cohorts of people. There appears to be overwhelming evidence that patients from certain minority ethnic groups have differential outcomes in disease severity and even death 1,2.

The review by Chakravorty published in this edition, explores the science behind this 'risk' and the rationale for stratification. 3 There are several theories critically explored in this review from biological to demographic and socio-economic variables. This phenomenon of differential outcomes to a similar degree is also seen in health workers, where ethnicity appears to be an independent risk, yet they are so fundamentally different from minority ethnic population groups, in terms of exposure and background.2 It is therefore inconceivable, that their (health professionals) experience in the face of COVID-19, can be rationalised in the same way. 
Although initially thought to be related to the impact of increased prevalence of comorbid conditions, 1 it now appears to also include non-biological factors and societal variations in how people are treated differently. This is not surprising to a significant minority, who are well aware of deeply ingrained discrimination in many societies, and the impact this has on their lives, livelihood and health outcomes. One hypothesis being considered is that 'structural discrimination' may have contributed to magnifying the adverse impact of COVID-19 in certain populations, 4 and therefore deserves a more centre stage in our public discourse, to help us determine cause and effect.

Why whole communities in different geographical cohorts have differential health outcomes is complicated, and perhaps dependent on multiple factors including biology, education, socio-economic status, culture, ease of access to facilities/ resources, language and organisational or even political attitudes.

This editorial cannot do complete justice to this wider question, but we wish to limit our exploration to the wisdom drawn from a lived-experience of a well-defined cohort of health workers in the United Kingdom who are at the forefront of the COVID-19 pandemic. We have drawn on the experience of a wide range of health professionals through personal contacts, social networks and reported analysis of anonymised surveys from across the health spectrum. 5 The aim of this dialogue is to raise the issues we feel are important in understanding the differential outcomes for health workers and encourage all stakeholders to urgently commission actions which will help to 'save lives and save (all within) the NHS'.

Esmail and Everington described the variation in success in interviews and career progression for Black, Asian and Minority Ethnic (BAME) students and doctors over the last 25 years. 6 In a commentary of their experience over two decades they concluded that raising awareness of such issues is not without its dangers; at one stage they were charged with misconduct by the General Medical Council. 6 Roger Kline, a research fellow at the University of Middlesex Business School, has been persistent in his work raising awareness of the differential access to care for patients, and outcomes for staff from BAME backgrounds in the NHS.7 In 2015, the Workplace Race Equality Standards (WRES) were established and NHS organisations were required to report on a range of metrics reflecting their culture of equality, diversity and fairness.8 The most recent report in 2019, shows improvement, but progress remains slow and the issues do not currently occupy enough bandwidth with senior leadership or the commissioners to speed up implementation. 9

\section{Why is it important to explore differential health outcomes for health workers from BAME backgrounds?}

The answer is probably in the emerging evidence of high risk of disease or death among health workers in the UK 10, 2, Southern Europe11 and USA. 12 Most alarmingly, both within the general population, and in health workers, the outcomes appear to be disproportionately worse in those from a BAME background. Early reviews of the risk factors from hospital admissions in the UK and USA, indicates that several factors relating to obesity, concurrent chronic diseases, demography and social deprivation may be important. However, in this pandemic up to $20 \%$ of health workers appear to be afflicted, a figure much higher than in Middle Eastern Respiratory Syndrome (MERS) or SARS, and have a higher risk of death or severe disease, than those identified in the population.11 All health workers due to the nature of their roles, naturally face a higher risk of exposure to SARS-CoV-2 in their line of duty. Yet the observed differential outcomes, segregated by ethnicity is difficult to explain by 'the exposure hypothesis' alone. There appears to be another missing factor ' $R$ '. In this pandemic, the letter ' $R o$ ' has seen more popularity than ever before, as politicians and citizens alike, have struggled to make sense of the reproduction 
number of the 'virus' in understanding transmission prevention strategies, previously an exclusive preserve of epidemiologists.

\section{What then, are the potential contributory factors specific to health workers?}

The best way to explore this would be to start with health workers and their perceptions of their own environment. Surveys by BAPIO Institute for Health Research5, British Medical Association13,14, the Royal College of Physicians 15 and a large study by Goldacre et al 16 all indicate that health workers are facing varying degrees of challenges in availability of personal protection equipment, ability to provide care while complying with social distancing and in their challenge of finding ways to avoid exposure, when vulnerable. 5 While there appears to be an unacceptably high variation in the availability of personal protective equipment (PPE) between clinical settings in hospital, primary care and care homes, this risk appears to be double in those from a BAME background. 17 There are huge variations in the proportions of workers from a BAME background in roles with lower pay and higher exposure, both in clinical and supportive roles. Often these are workers employed by agencies contracted to the NHS and local councils and may not have the infrastructure to provide a comprehensive occupational health risk assessment and support. There is evidence that hospitals were prioritised by the NHS distribution networks for PPE, perhaps erroneously assuming that the risk in care homes or primary care settings was lower. The results of such policy decisions may be manifest in the high rate of overall deaths recorded in the UK and USA due to COVID-19, including care homes18, when compared to China, South Korea, Germany, South Africa, New Zealand and Australia. As the realisation of the higher rate of disease and death among health workers has come, there have been multiple appeals by organisations representing health workers to institute risk assessment and enhanced protection-avoidance strategies. 19

BAPIO has joined with BMA, Royal Colleges and other bodies to seek urgent action. 20 Public Health England and NHS England/Improvement have been working with public health experts to develop and implement such tools. Many large and progressive NHS organisations with strong BAME liaison networks have been quick to listen to the concerns of their staff and developed tools themselves. $21 \mathrm{~A}$ review of such tools undertaken by BIHR researchers as published in this journal and others 3 suggest that the methodology appears to be simply based on biological factors with a composite weightage added for 'ethnicity'.22 This appears to be a fundamental flaw in the understanding of the vast range of potential issues which unite health workers from a diverse social, professional, educational, cultural and religious backgrounds who are in the 'BAME' category. It is unfathomable why such a diverse group of professionals will have the same experience and what might be the factor uniting them to have the same 'statistical weightage' in the eyes of the novel coronavirus SARS-CoV-2.

Shilpa Ross, writing for the Kings Fund report 'We're here and you're there': lived experiences of ethnic minority staff in the NHS' 23 describes a compilation of stories of microaggressions, exclusion, differential treatment, stunted career progression, anxiety and even fear. There are reports of international health workers from across the world having difficulties in obtaining support for family members, having to pay additional tariff for access to health and restriction to changing their jobs due to Home Office regulations. In all reports of health workers being afflicted by COVID-19 there is no information on their residence/ immigration status, but it is conceivable that a large proportion of health workers in the frontline are facing such personal challenges.

What impact does this insecurity have on the choices they make at work? Is it possible that such insecurities may stunt one's ability to raise concerns or challenge unfair treatment? Anecdotes and 
personal stories from many BAME staff across the country seems to suggest that there is a perceived link between the inequality rife in some organisations and the outcome in the face of COVID-19.

NHS England is committed to meeting the WRES targets, and most senior leaders have pledged their support for equality for their patients and staff as well as valuing the richness of diversity. There is much more work to be done to get this message to the middle level of management and to leaders on the frontline. It is time for the scientific community to dissect and explore the multitude of risks emboldened within the 'ethnicity' factor in an objective as well as qualitative way. It is time for BAME leaders to raise awareness of the particular risks to their communities and support their members to come forward to join the BAME liaison committees being formed in different organisations, talk openly about the 'their lived-experience' and help their colleagues and middle-level leaders to take urgent mitigating actions, which are culturally appropriate. Too many lives have been lost perhaps inadvertently, in this pandemic. SARS-CoV-2 has rather dramatically shone the light on the social, health and economic determinants of outcomes in the general population and staff alike. This is a call for action and for ongoing research to explore this area. Science has been underpinning the strategy of many governments during the COVID19 pandemic and it is imperative that 'science has to show the way' in mitigating the risk for BAME doctors facing adverse health outcomes, simply by the nature of their ethnic background.

Another important issue is the inhomogeneity among the cohorts who constitute the BAME population. For example, the umbrella term. "Asian" includes South East Asian, Chinese, and South Asians. Even among people from the Indian sub-continent, there are significant social, and cultural differences between people originating from India, Pakistan, Bangladesh, and Sri Lanka. These differences and their impact on health outcomes need to be explored in more detail, to be able to understand better, and mitigate the impact of differential outcomes in the BAME population.

\section{References}

1. CDC. Coronavirus Disease 2019 (COVID-19). Centers for Disease Control and Prevention https://www.cdc.gov/coronavirus/2019-ncov/need-extra-precautions/racial-ethnic-minorities.html (2020).

2. Cook, T., Kursumovic, E. \& April 2020, S. L. Exclusive: deaths of NHS staff from covid-19 analysed. Health Service Journal https://www.hsj.co.uk/exclusive-deaths-of-nhs-staff-from-covid-19analysed/7027471.article.

3. Chakravorty, S. Risk Stratification in COVID-19: A Review. The Physician 6, (2020). DOI: https://doi.org/10.38192/1.6.1.11

4. Devakumar, D., Shannon, G., Bhopal, S. S. \& Abubakar, I. Racism and discrimination in COVID-19 responses. The Lancet 395, 1194 (2020).

5. Chakravorty, I. et al. An Online Survey of Healthcare Professionals in the COVID-19 Pandemic in the UK: : SUSHRUTA J. Health Policy Opin. 13, (2020). DOI https://doi.org/10.38192/13.2.9

6. Aneez Esmail and Sam Everington: The perils of researching racial discrimination. The BMJ https://blogs.bmj.com/bmj/2020/02/13/aneez-esmail-and-sam-everington-the-perils-ofresearching-racial-discrimination/ (2020).

7. Racism in the NHS: don't let the unspeakable become acceptable. openDemocracy https://www.opendemocracy.net/en/ournhs/racism-in-nhs-don-t-let-unspeakable-becomeacceptable/.

8. NHS England » NHS Workforce Race Equality Standard. https://www.england.nhs.uk/about/equality/equality-hub/equality-standard/.

9. NHS England » Workforce Race Equality Standard data reporting - 2019. 
https://www.england.nhs.uk/publication/workforce-race-equality-standard-data-reporting-2019/.

10. Hunter, E. et al. First experience of COVID-19 screening of health-care workers in England. The Lancet 395, e77-e78 (2020).

11. Sim, M. R. The COVID-19 pandemic: major risks to health and other workers on the front line. Occup. Environ. Med. 77, 281-282 (2020).

12. CDCMMWR. Characteristics of Health Care Personnel with COVID-19 - United States, February 12April 9, 2020. MMWR Morb. Mortal. Wkly. Rep. 69, (2020).

13. Rimmer, A. Covid-19: Two thirds of healthcare workers who have died were from ethnic minorities. BMJ 369, (2020).

14. Scavone, F. Most doctors still lack protective equipment, finds survey. The British Medical Association is the trade union and professional body for doctors in the UK.

https://www.bma.org.uk/news-and-opinion/most-doctors-still-lack-protective-equipment-findssurvey-1.

15. COVID-19 and its impact on NHS workforce. RCP London https://www.rcplondon.ac.uk/news/covid19-and-its-impact-nhs-workforce (2020).

16. Collaborative, T. O. et al. OpenSAFELY: factors associated with COVID-19-related hospital death in the linked electronic health records of 17 million adult NHS patients. medRxiv 2020.05.06.20092999 (2020) doi:10.1101/2020.05.06.20092999.

17. Daga, S. et al. Self-reported Occupational Risk for COVID-19 in Hospital Doctors from Black Asian \& Minority Ethnic Communities in UK. The Physician 6, (2020). DOI https://doi.org/10.38192/1.6.1.9

18. correspondent, R. B. S. affairs. Half of coronavirus deaths happen in care homes, data from EU suggests. The Guardian (2020).

19. Organization, W. H. Health workers exposure risk assessment and management in the context of COVID-19 virus: interim guidance, 4 March 2020. (2020).

20. BAPIO sends letter to NHS employers regarding COVID-19: Disproportionate high mortality rates in BAME health and social care (HSCW) workers. BAPIO Training Academy (BTA) https://www.bapiotrainingacademy.com/bapio-sends-letter-to-nhs-employers-regarding-covid-19disproportionate-high-mortality-rates-in-bame-health-and-social-care-hscw-workers/ (2020).

21. NHS trust prioritises BAME staff over Covid-19. BBC News (2020).

22. Individual Staff Risk Assessment Checklist for Covid-19. 5.

23. 'We're here and you're there': lived experiences of ethnic minority staff in the NHS. The King's Fund https://www.kingsfund.org.uk/blog/2019/11/lived-experiences-ethnic-minority-staff-nhs (2019). 